\title{
Diamond Rib: A Mechanical Design Method for Improving the Stiffness of a Structure
}

\author{
Kazunori Shinohara1, Masanobu Mizoguchi' ${ }^{1}$, Shintatsu Suzuki1, Yasutaka Narusawa ${ }^{2}$ \\ ${ }^{1}$ Department of Integrated Mechanical Engineering, Daido University, Nagoya, Japan \\ ${ }^{2}$ Collaborative Research Center, Daido University, Nagoya, Japan \\ Email: shinohara@06.alumni.u-tokyo.ac.jp
}

Received 28 April 2016; accepted 19 June 2016; published 22 June 2016

Copyright (C) 2016 by authors and Scientific Research Publishing Inc.

This work is licensed under the Creative Commons Attribution International License (CC BY). http://creativecommons.org/licenses/by/4.0/

(c) (i) Open Access

\begin{abstract}
It is necessary to decrease the weight of space satellites, while maintaining their stiffness. To achieve this weight reduction, many structures, such as honeycomb and isogrid, have been reported in the literature. In this paper, the diamond rib method, a mechanical design method for improving the stiffness of structures, is introduced. By applying this method to a small space satellite, we propose a new structure called the "Diamond Rib Structure". This structure significantly improves the ability to withstand the vibrational disturbances in a rocket fairing.
\end{abstract}

\section{Keywords}

Space Satellite, Finite Element Method, Optimal Design, Natural Frequency, Natural Mode, Diamond Rib Method, Stiffness

\section{Introduction}

ChubuSat [1] [2] is a small, 50-kg-class satellite as shown in Figure 1. It is developed by a consortium of universities and aerospace companies such as MASTT [3], Nagoya University [2], and Daido University [4]. MASTT (Monozukuri Aerospace Support Technology Team) was established by Japanese manufacturers. By decreasing the cost of the space satellite, ChubuSat, we promote the participation of private companies in outer space business ventures.

The rocket launch is shown in Figure 2. The combustion vibrations and acoustic vibrations of a rocket engine cause the disturbance at the time of rocket launch. Irregular vibration consisting of different vibration frequency bands occurs in rocket fairing. In particular, powerful disturbances of low frequency transient take place around $5-100 \mathrm{~Hz}$ at the rocket launch [5]. Various vibration modes of a space satellite such as ChubuSat are excited over a wide range from a low-frequency to a high frequency, triggering the disturbance in the fairing. On the 


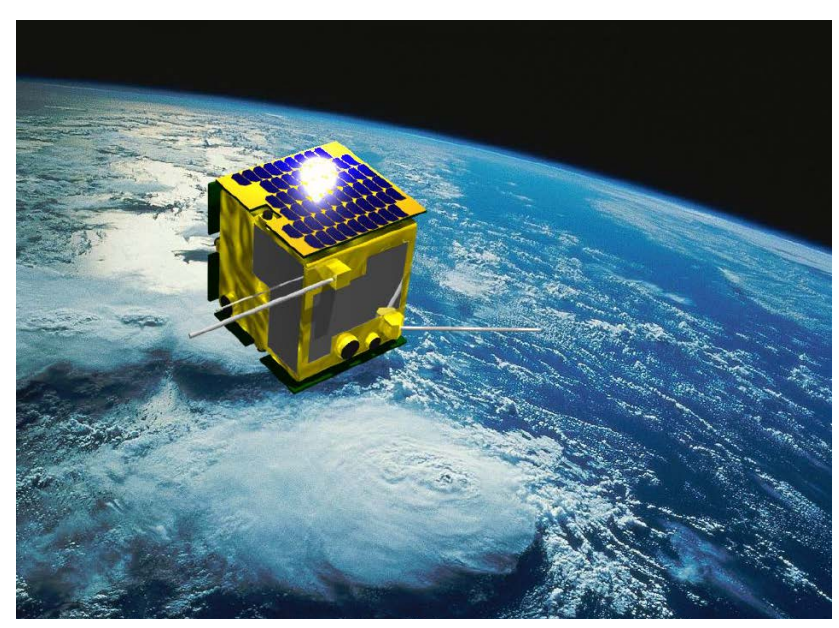

Figure 1. Space satellite: ChubuSat [1] [2] (Image credit: Nagoya university [2]).

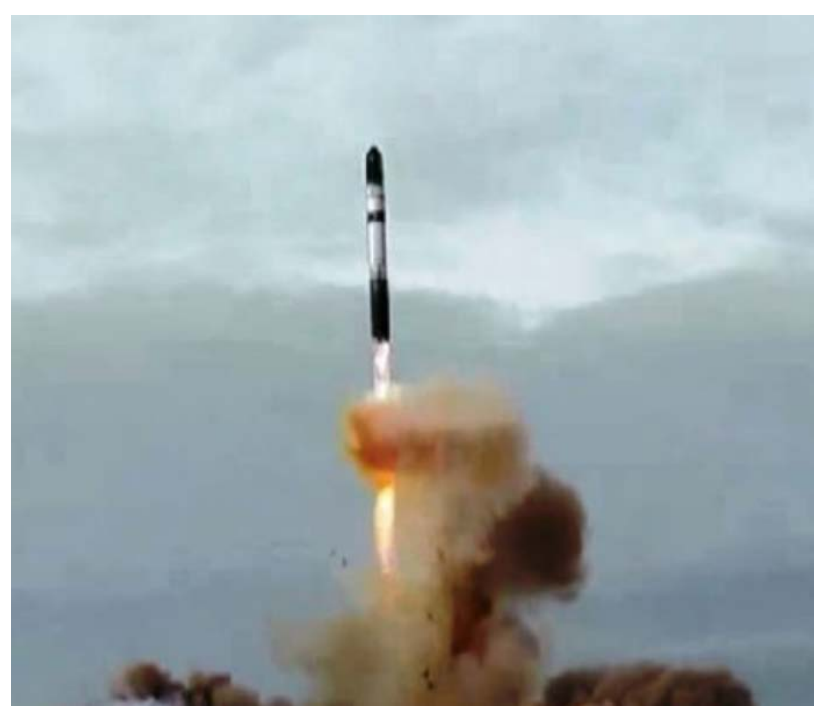

Figure 2. Dnepr Rocket Launch (as the piggyback microsatellite; the launch of ChubuSat was successfully performed from Yasny Launch Base, Orenburg region, Russia, on November 6, 2014) (Image credit: ISC Kosmotras [16]).

other hand, the low natural frequency of the satellite depends on the satellite structure and the connection parts between the rocket fairing and the satellite. In particular, the low natural frequency of a small space satellite occurs around $100 \mathrm{~Hz}$. The disturbance vibration frequency attributable to vibrations such as combustion vibration is close to the low natural frequency of the satellite. Induced by two disturbances with different frequencies, the total disturbance tends to resonate. Because of resonance, the entire satellite vibrates severely. The resonance may cause some damage to the observation equipment located on the sidewall of the satellite structure. Some damage may occur in the form of connection failures in electrical and electronic devices mounted on the observation system. Because of such damage, the satellite may fail to observe natural phenomenon such as natural disasters or severe weather events even if it can successfully position itself into orbit. The stiffness of the structure of a satellite should be increased so that the low-order natural frequency is just above $100 \mathrm{~Hz}$. To avoid resonance and to increase the stiffness, the structures are generally reinforced by adding new mechanical elements such as ribs. Due to the weight limitation of a space satellite mounted on a launch rocket, this approach of reinforcing the satellite by adding mechanical elements is not acceptable because it increases the launch weight.

Previous studies have indicated that to maintain the reliability of satellite structures and to reduce the vibra- 
tions, sheet panels can be added on the surface of the structures. The weight of the sheet panels can be considered almost zero (See Ref. [6]). In Reference [7], in order to avoid vibrations, the elastic members can be attached to the gap between the panels. In Reference [8], the specified natural frequency was reduced by installing a spring in the connecting parts between the satellite and rocket fairing. In Reference [9], adhesive having vibration-damping effects was used to bond the metal parts of a sandwich panel. In Reference [10], the characteristics of vibration damping were improved by inserting viscoelastic damping pads at the hinge parts to expand the panel. In Reference [11], at the time of rocket launch, the vibration was suppressed by adopting a vibration control system using both sensors and actuators. In Reference [12], the natural frequency can be adjusted by installing cushioning plate springs. To improve the stiffness, an isogrid structure [13] as shown in Figure 3 or a honeycomb structure as shown in Figure 4, etc. were proposed in reference. Using the effect of the second moment of the area, the structure can be reinforced more than ever, while the weight of the structure can be reduced. Hollow triangular ribs are regularly arranged on a flat plate. The ribs of the isogrid structure are uniformly arranged on the plate. An excessive number of ribs are added in the local area. In order to minimize the satellite

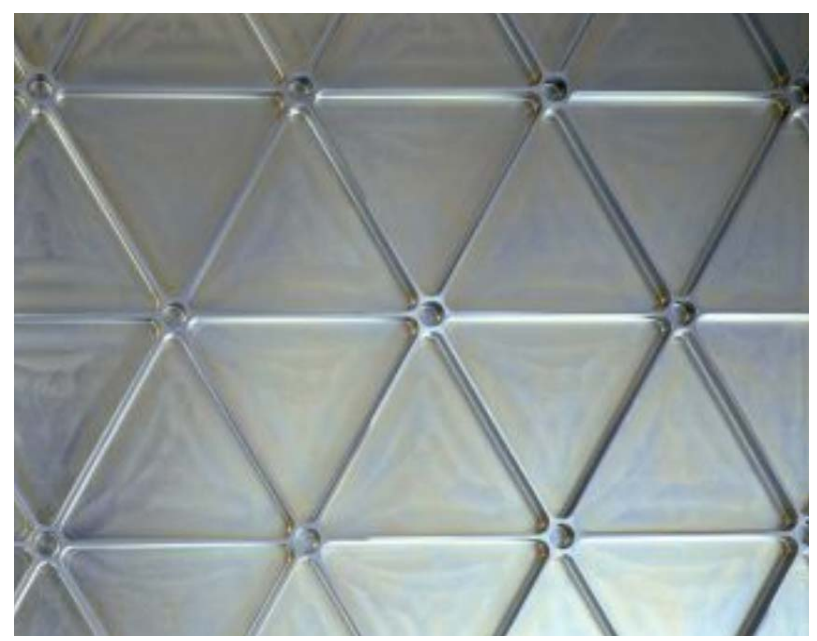

Figure 3. Isogrid structure (image credit: JAXA [17]).

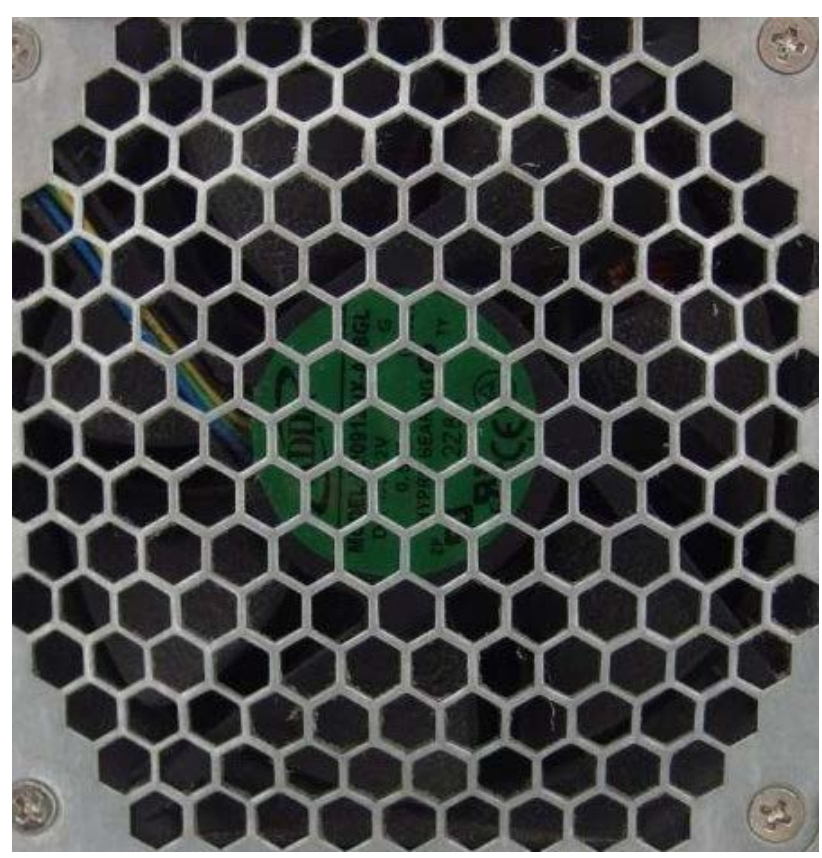

Figure 4. Honeycomb structure. 
weight, it is necessary to optimize the arrangement of the ribs. To maximize the objective function, an optimum design method was applied to the shape of the structure. Optimum design methods generally consist of sizing optimization, shape optimization and topology optimization [14] [15]. In sizing optimization, the design variables are applied as the geometrical parameters such as the representative length, width or thickness, etc. These parameters are optimized by modifying the CAD model. We can obtain the value of the objective function by analyzing the results. By deforming the shape of the structure, the algorithm based on shape optimization searches for the optimum value of the objective function. In topology optimization, the algorithm searches for the forms of structures in addition to deformation in the shapes of structures. The forms of structures are varied by creating forms such as gaps and holes in structures and by deforming the shapes of structures.

In this paper, the diamond rib method is described. This method is a type of sizing optimization. Using the method, the optimal panel, the diamond rib structure, was created. Aiming to develop a robust satellite, the diamond rib structure is applied to a 50-kg small satellite in Figure 5. Thought the results of a 50-kg small satellite, the effect of the vibration suppression is verified.

\section{Problem of Small Space Satellites}

To estimate the natural frequency of a satellite structure, $\omega$, the following equation can be used:

$$
\omega=\sqrt{\frac{k}{m}}
$$

The variables $m$ and $k$ represent the mass and spring constant, respectively. In the case of a plate, the natural frequency, $\omega$, can be obtained based on material properties such as the Young's modulus and the second moment of the area. The natural mode of a panel in a 50-kg class small satellite is shown in Figure 6. The antinode of the natural mode with respect to a low natural frequency is formed at the center of the plate. The space satellite falls into a resonant state if the frequency of the disturbance is close to the natural frequency of the space satellite, where upon the satellite vibrates in a violent manner. As a result, the observation equipment or the satellite structure may be damaged. The disturbance frequency is around $100 \mathrm{~Hz}$. If the natural frequency of the satellite structure is above $100 \mathrm{~Hz}$, the reliability of the vibration design can be improved by avoiding resonance. At least, the natural frequency for one of the panels in the satellite should be over $400 \mathrm{~Hz}$, as shown in Figure 6. As described in Ref. [6]-[12], to achieve this, reinforcing parts or vibration control devices are generally attached to the satellite.
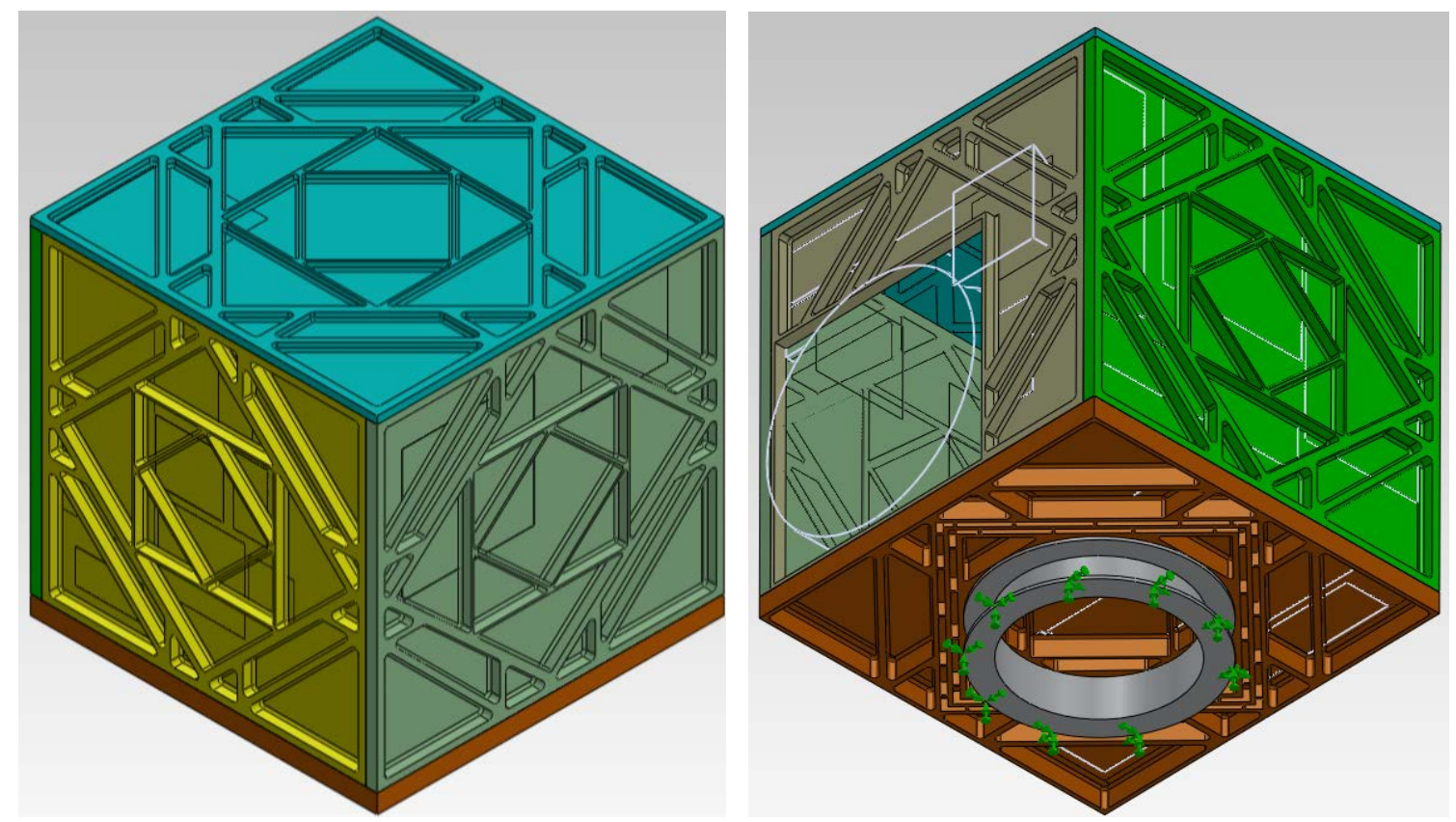

Figure 5. A small space satellite using optimal panels (diamond rib structures). 


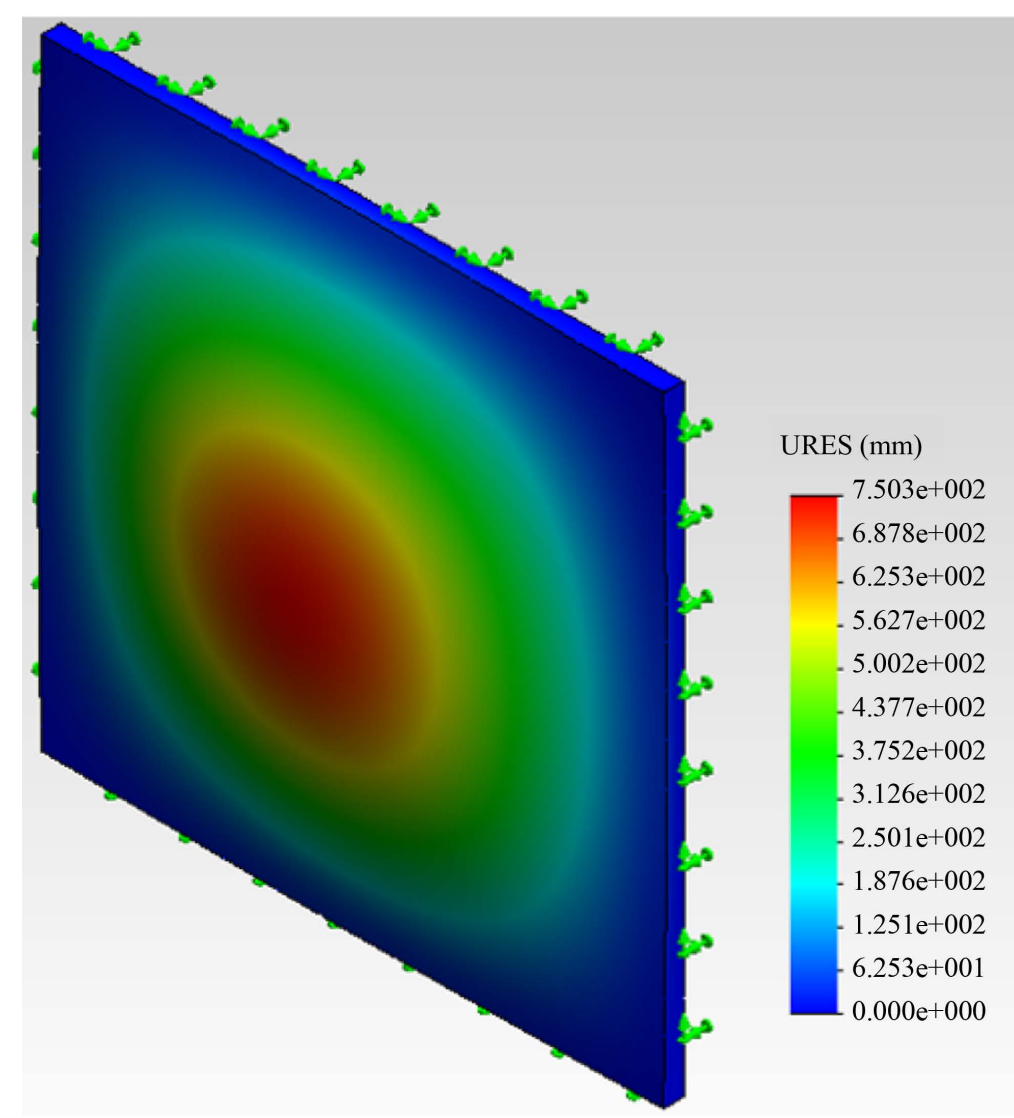

Figure 6. Natural mode with respect to one side panel (natural frequency: 479 $\mathrm{Hz}$, mass: $8506 \mathrm{~g})$.

\section{Principal of Diamond Rib Method}

Various natural modes exist for each plate. By visualizing natural modes through simulation results, we can confirm the occurrence of antinodes or nodes at local areas for each plate. Deformation patterns of the natural vibration mode can be predicted by using the simulation technology. In general, the ribs of the structure were cut out from one plate so that the ribs were set with respect to the antinode of natural modes. However, if inserting ribs as shown in Figure 7, ribs can't work to increase the natural frequency. On the other hand, the natural frequency becomes seven lower with the addition of unnecessary ribs. The added ribs can also be regarded as added mass, $m$, in Equation (1), but are not responsible for stiffness. In this method, these ribs suppress the circumference of the antinode on the flat plate, but not at the top of the antinode on the flat plate. With respect to the area where the antinode occurs on the flat plate, the stiffness cannot be increased by adding ribs. To decrease the thickness around the center of the flat plate, the center of one flat plate was cut more deeply than the circumference of the flat plate. The natural frequency turned out to be higher with this approach.

The algorithm based on this method is shown in Figure 8. In this paper, the diamond rib method and the diamond rib structure are described. The diamond rib method is a design method for imparting high stiffness. The algorithm based on the method searches for the optimal structure under the given constraint conditions (mass, etc.). The diamond rib structure is the structure constructed by the diamond rib method. The approaches of the optimal design are roughly classified in three groups. They are sizing optimization, shape optimization, and topology optimization. This method is one of the sizing optimizations. The vibration modes in a design problem are concentric, as shown in Figure 6. These modes occur at low frequencies. The geometric correlation between the concentric vibration mode and the diamond rib is low. Using the ribs of the low correlation geometrically, the vibration can effectively be suppressed. This rib arrangement is referred to as a primary diamond arrangement.

A primary diamond arrangement was rotated from $0^{\circ}$ to $45^{\circ}$. The secondary diamond arrangement was created 


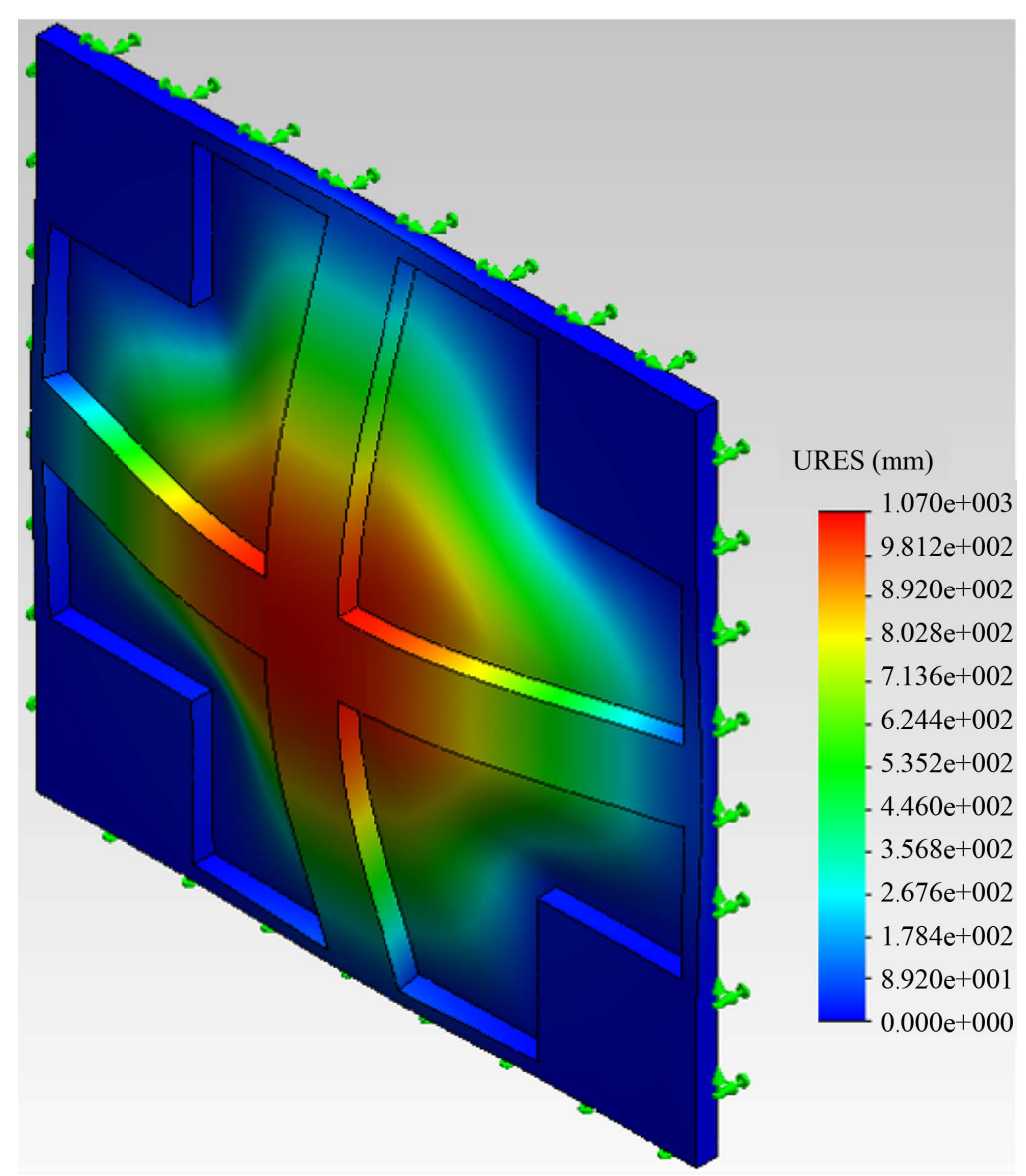

Figure 7. Natural mode with respect to one side panel with ribs (natural frequency: $308 \mathrm{~Hz}$, mass: $4300 \mathrm{~g}$ ).

by rotating the primary diamond arrangement. As inscribed in the primary diamond rib arrangement, the secondary diamond arrangement was obtained. As mentioned above, the procedure of diamond rib arrangement was repeated in the same way. Therefore, the diamond rib arrangements consisted of the primary diamond rib arrangement, the secondary diamond rib arrangement, the tertiary diamond rib arrangement, etc. Using the results of vibration analysis, we can find that some diamond arrangements cannot contribute to the increase in the natural frequency of the structure. The algorithm is terminated after removing a diamond arrangement that does not contribute to the increase in the natural frequency. In this method, the initial structure achieves to the optimal structure. In the paper, the optimal structure is referred to as the diamond rib structure.

As stated above, although this is a computer simulation method, it is possible to create a real panel. Using one flat plate, the diamond rib structure can be cut out using a cutting machine. The diamond rib structure does not require the use of special techniques such as welding technologies. A small space satellite can easily be manufactured with bolted panels. With the aim of developing a commercialized satellite, the diamond rib method can also contribute to cost reduction.

\section{Algorithm of Diamond Rib Method}

The algorithm of the diamond rib method is shown in Figure 9.

(Step 1) The $3 D$ model of one panel in a space satellite is created using a software.

(Step 2) To calculate the $3 D$ model by the finite element method, the $3 D$ model is divided into meshes. The material properties, boundary conditions, and initial condition are inputted into the $3 D$ model.

(Step 3) The natural frequency and natural mode are calculated using a CAE software.

(Step 4) The location of the antinode is confirmed by visualizing the natural frequency. 


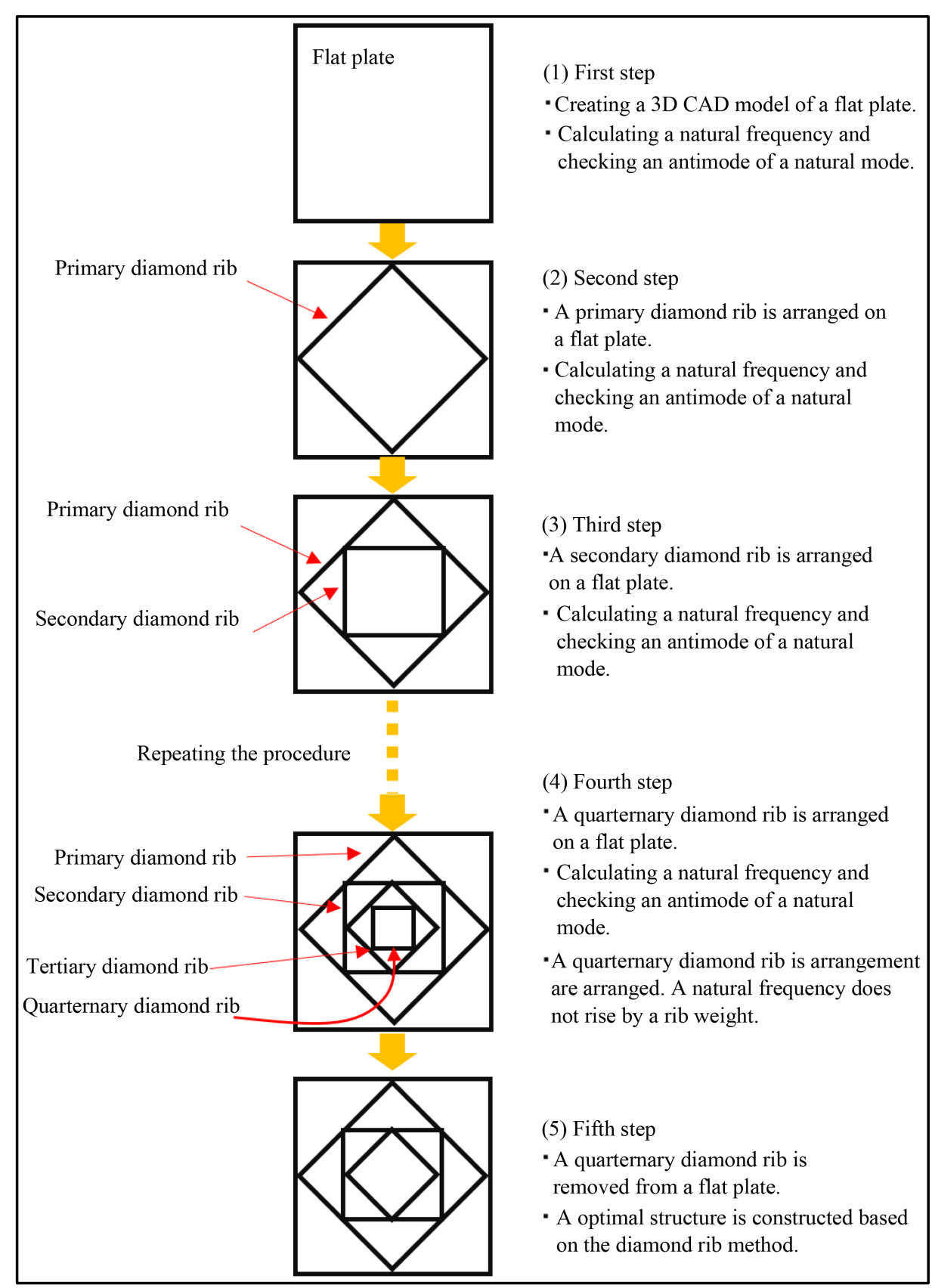

Figure 8. Rib arrangement based on the diamond rib method.

(Step 5) The antinode of the natural mode is surrounded by diamond ribs. The diamond rib arrangement is referred to as the primary diamond rib arrangement.

(Step 6) Using the $3 D$ model with the primary diamond rib arrangement, natural frequencies and natural modes are again calculated using a CAE software.

(Step 7) As inscribed in the primary diamond rib arrangement, the secondary diamond arrangement is obtained. Using the modified $3 D$ model, the natural frequency and mode are again calculated. Steps 5 to 7 are repeated until the natural frequency increases.

(Step 8) Based on the calculation results, if the $n^{\text {th }}$ diamond rib arrangements do not contribute to the increase in the natural frequency, these ribs are removed from the panel.

(Step 9) The panels based on the $n-1^{\text {th }}$ diamond rib arrangements are implemented in the space satellite. 


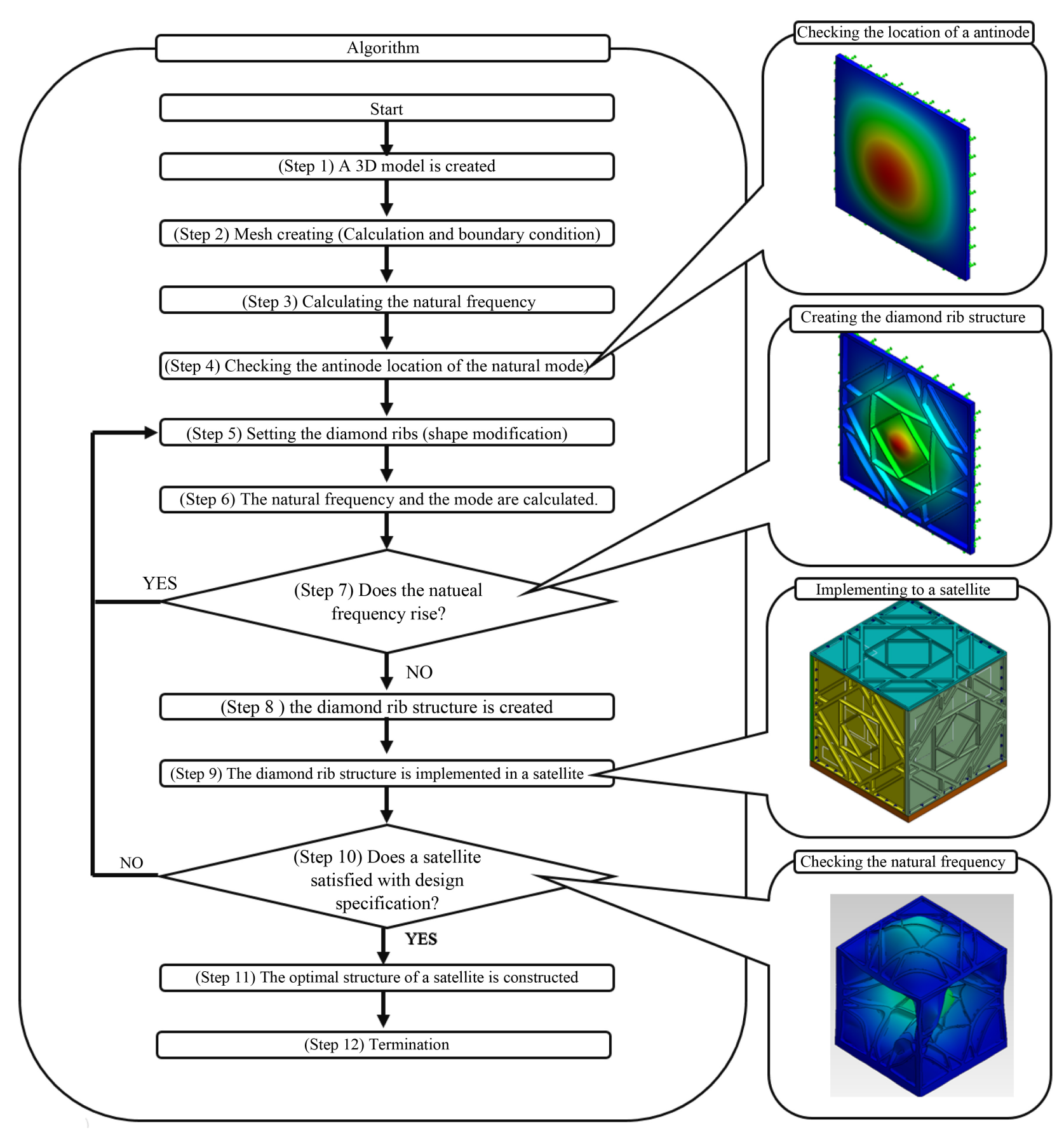

Figure 9. Algorithm based on the diamond rib method.

(Step 10) Using the space satellite $3 D$ model, the natural frequency and mode are calculated. We confirm that the natural frequency is over $100 \mathrm{~Hz}$.

(Step 11) If the natural frequency is not more than $100 \mathrm{~Hz}$, the high and the width of the ribs are varied by increasing the second moment of area or decreasing the weight.

(Step 12) The algorithm is terminated.

\section{Effect of Diamond Rib Method}

The optimal panel, based on the diamond rib method, is shown in Figure 10. In the optimal panel, the natural 

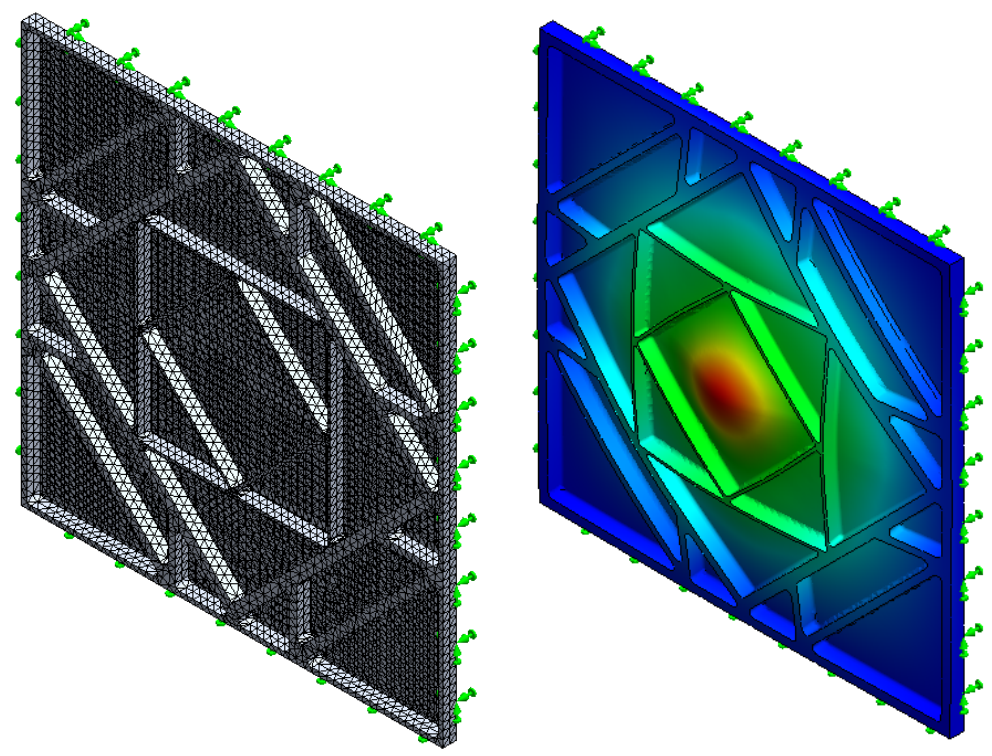

Figure 10. Mesh and natural mode of the diamond rib structure (natural frequency: $412 \mathrm{~Hz}$, mass: $1906 \mathrm{~g}$ ).

frequency and mass are $412 \mathrm{~Hz}$ and $1906 \mathrm{~g}$, respectively. Compared with the mass of the flat panel, the mass of the optimal panel can be reduced by about $25 \%$. On the other hand, the natural frequency $(479 \mathrm{~Hz})$ of the flat panel in Figure 6 almost matches that of the optimal panel $(412 \mathrm{~Hz})$, as shown in Figure 10. Thus, with the optimal panel, the reduction in natural frequency is not because of a decrease in mass and stiffness.

The reinforced panel with ribs is shown in Figure 7. With respect to the location of the antinode, the ribs are directly arranged on the flat panel. The antinode of the natural mode is suppressed. Using this approach, the mass decreases to about half of the mass of the flat panel in Figure 6. On the other hand, the natural frequency is also reduced by about $25 \%$ compared with the natural frequency of the flat panel. Therefore, the ribs on the plate, as shown in Figure 7, are not effective compared with the ribs in Figure 10.

Using the optimal panel, the natural vibration and mode of a small satellite model are calculated. The natural mode of a small space satellite is shown in Figure 11 and Figure 12. The first, second, and third modes depend on the stiffness of the support ring. Using the ring, the satellite is fixed to the rocket fairing. The natural mode of the vibration design problem at the time of launch rocket is the fourth natural mode. The fourth natural frequency is $412 \mathrm{~Hz}$. Using the five optimal panels (the diamond ribs structure), the mass of the satellite can be reduced by about $33,005 \mathrm{~g}(=8507(\mathrm{~g}) \times 5-1906(\mathrm{~g}) \times 5)$, compared with the satellite using the five flat panels. The natural mode of the satellite with five optimal panels is $255.5 \mathrm{~Hz}$. The design specification of a natural frequency of more than $100 \mathrm{~Hz}$ in the vibration design problem at the time of launch rocket is satisfied.

\section{Conclusions}

In this paper, the diamond rib method was introduced and described. After checking the antinode pattern of the natural mode, the ribs pattern on the flat panel was arranged based on the antinode pattern. The ribs pattern was called the diamond rib structure. The diamond rib structure was applied to a small space satellite.

The conclusions are summarized as follows:

- Compared with satellite structures with a flat plate, the mass of a small space satellite is reduced by about $25 \%$, while the natural frequency of the optimal satellite almost matches the original design value for the small space satellite.

- Special techniques, such as a welding technology, are not needed in the approach based on the diamond rib method. Therefore, it is possible to reduce the manufacturing cost of the small space satellite.

The diamond rib method was applied to the flat panel. The diamond rib method can also be applied to various other complex structures, only if the antinode of natural frequencies can be obtained by using a CAE software.

As the final goal, the diamond rib structure will be implemented in the $50-\mathrm{kg}$ class satellite: ChubuSat. 


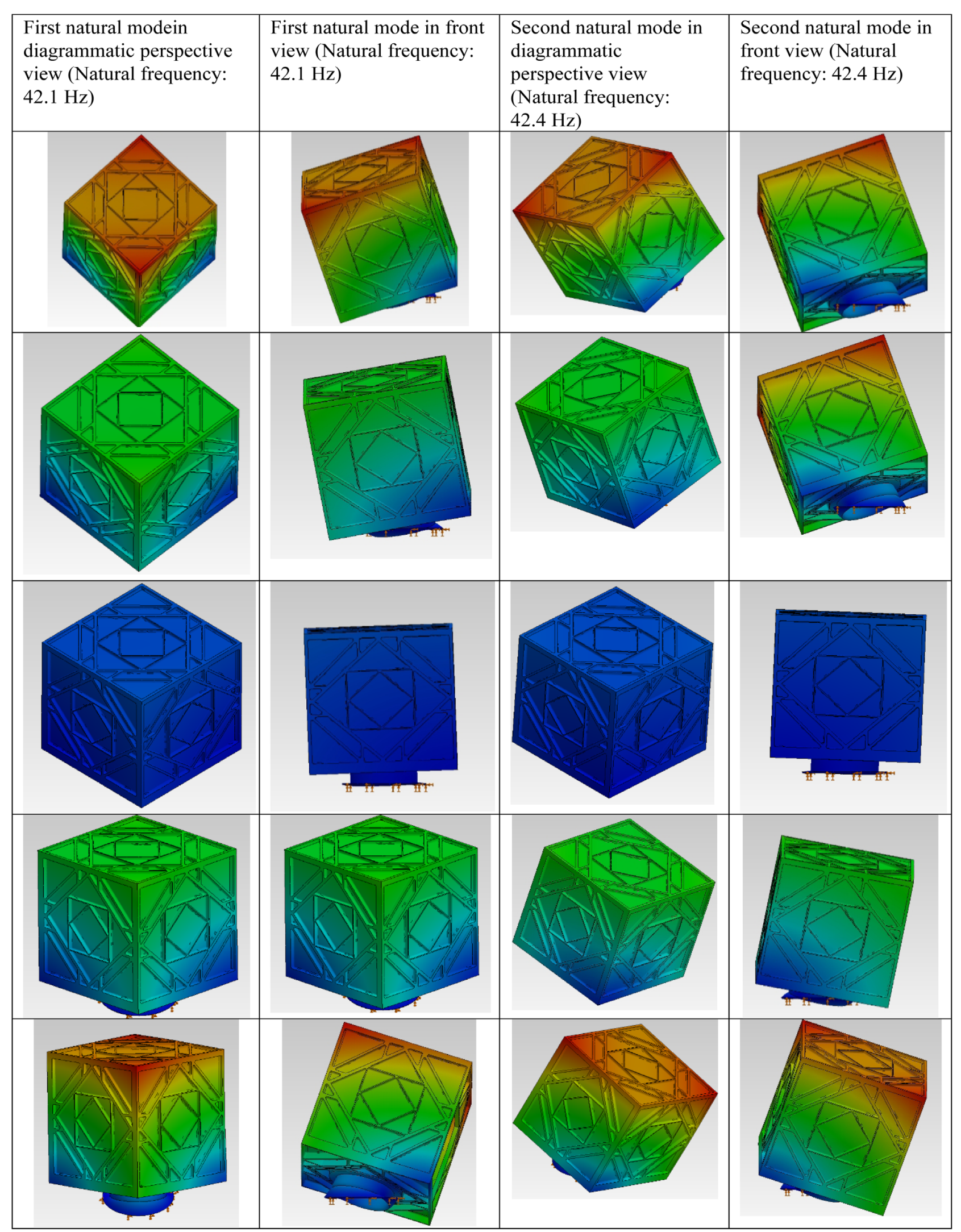

Figure 11. The first and second natural modes of a small space satellite. 


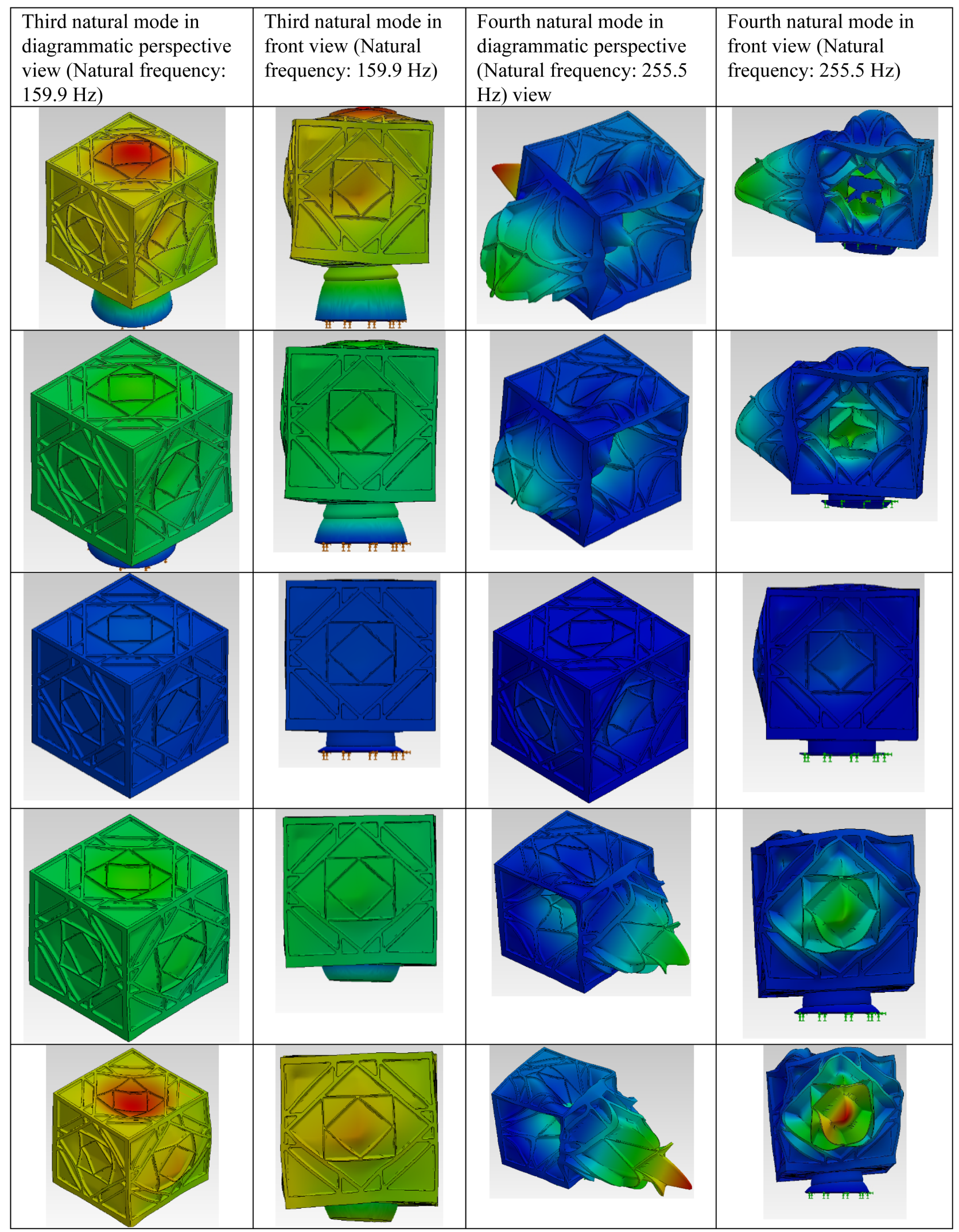

Figure 12. The third and fourth natural modes of a small space satellite. 


\section{References}

[1] ChubuSat. https://ja.wikipedia.org/wiki/ChubuSat

[2] Nagoya University. https://www.frontier.phys.nagoya-u.ac.jp/index.html

[3] MASTT. http://www.mastt.jp/

[4] Daido University. http://www.daido-it.ac.jp/english/

[5] Wijker, J. (2004) Mechanical Vibrations in Spacecraft Design. Springer, Berlin, Heidelberg. http://dx.doi.org/10.1007/978-3-662-08587-5

[6] Steven, H. and Ozaki, T. (2002) Japan Patent Kokai. 2002-353489.

[7] Ozawa,T. and Ozaki, T. (2003) Japan Patent Kokai. 2003-267299.

[8] Takahashi, A. (2000) Japan Patent Kokai. 2000-006900.

[9] Utsunomiya, S. (2000) Japan Patent Kokai. 2000-128094.

[10] Okudaira, T. (1998) Japan Patent Kokai. 1998-236400.

[11] Kabashima, S. and Ozaki, T. (2003) Japan Patent Kokai. 2003-278826.

[12] Douke, M. and Higashikata, H. (1993) Japanese Unexamined Patent Application Publication. 1993-27385.

[13] Meyer, R.R., Harwood, O.P. and Orlando, J.I. (1973) Isogrid Design Handbook. NASA-CR-124075, NASA, Center for Aerospace Information (CASI).

[14] Ma, Z.-D., Kikuchi, N. and Hagiwara, I. (1993) Structural Topology and Shape Optimization for a Frequency Response Problem. Computational Mechanics, 13, 157-174. http://dx.doi.org/10.1007/BF00370133

[15] Zhou, M., Pagaldipti, N., Shyy, Y.K. and Thomas, H.L. (2004) An Integrated Approach for Topology, Sizing, and Shape. Structural and Multidisciplinary Optimization, 26, 308-317. http://dx.doi.org/10.1007/s00158-003-0351-2

[16] ISC Kosmotras. http://www.kosmotras.ru/

[17] JAXA. http://www.jaxa.jp/ 